\title{
Hyers-Ulam Stability of a Generalized Second-Order Nonlinear Differential Equation
}

\author{
Maher Nazmi Qarawani \\ Department of Mathematics, AlQuds Open University, Ramallah, Palestine \\ Email: mkerawani@qou.edu
}

Received August 24, 2012; revised September 27, 2012; accepted October 4, 2012

\begin{abstract}
In this paper we have established the stability of a generalized nonlinear second-order differential equation in the sense of Hyers and Ulam. We also have proved the Hyers-Ulam stability of Emden-Fowler type equation with initial conditions.
\end{abstract}

Keywords: Nonlinear Differential Equation; Hyers-Ulam Stability; Emden-Fowler; Second-Order

\section{Introduction}

In 1940 Ulam posed the basic problem of the stability of functional equations: Give conditions in order for a linear mapping near an approximately linear mapping to exist [1]. The problem for approximately additive mappings, on Banach spaces, was solved by Hyers [2]. The result obtained by Hyers was generalized by Rassias [3].

After then, many mathematicians have extensively investigated the stability problems of functional equations (see [4-6]).

Alsina and Ger [7] were the first mathematicians who investigated the Hyers-Ulam stability of the differential equation $g^{\prime}=g$. They proved that if a differentiable function $y: I \rightarrow R$ satisfies $\left|y^{\prime}-y\right| \leq \varepsilon$ for all $t \in I$, then there exists a differentiable function $g: I \rightarrow R$ satisfying $g^{\prime}(t)=g(t)$ for any $t \in I$ such that $|g-y| \leq 3 \varepsilon$, for all $t \in I$. This result of alsina and Ger has been generalized by Takahasi et al [8] to the case of the complex Banach space valued differential equation $y^{\prime}=\lambda y$.

Furthermore, the results of Hyers-Ulam stability of differential equations of first order were also generalized by Miura et al. [9], Jung [10] and Wang et al. [11].

Motivation of this study comes from the work of $\mathrm{Li}$ [12] where he established the stability of linear differential equation of second order in the sense of the Hyers and Ulam $y^{\prime \prime}=\lambda y$. Li and Shen [13] proved the stability of nonhomogeneous linear differential equation of second order in the sense of the Hyers and Ulam $y^{\prime \prime}+p(x) y^{\prime}+q(x) y+r(x)=0$, while Gavruta et al. [14] proved the Hyers-Ulam stability of the equation $y^{\prime \prime}+\beta(x) y=0$ with boundary and initial conditions.

The author in his study [15] estabilshed the Hyers-
Ulam stability of the equations of the second order

$$
\begin{aligned}
& z^{\prime \prime}+p(x) z^{\prime}+q(x) z \\
& =h(x)|z|^{\beta} \mathrm{e}^{\left(\frac{\beta-1}{2}\right) \int p(x) \mathrm{d} x} \operatorname{sgn} z, \beta \in(0,1)
\end{aligned}
$$

and

$$
z^{\prime \prime}+p(x) z^{\prime}+(q(x)-\alpha(x)) z=0
$$

with the initial conditions $z\left(x_{0}\right)=0=z^{\prime}\left(x_{0}\right)$.

In this paper we investigate the Hyers-Ulam stability of the following nonlinear differential equation of second order

$$
z^{\prime \prime}-F(x, z(x))=0
$$

with the initial condition

$$
z(a)=z^{\prime}(a)=0
$$

where

$$
\begin{aligned}
& z \in C^{2}(I), I=[a, b],|F(x, z(x))| \leq A|z|^{\alpha}, \\
& \alpha>0,-\infty<a<b<\infty,-\infty<z<\infty, \text { and } F(x, 0)=0 .
\end{aligned}
$$

Moreover we investigate the Hyers-Ulam stability of the Emden-Fowler nonlinear differential equation of second order

$$
z^{\prime \prime}-h(x)|z|^{\alpha} \operatorname{sgn} z=0
$$

with the initial condition

$$
z(a)=z^{\prime}(a)=0
$$

where

$$
z \in C^{2}(I), I=[a, b],-\infty<a<b<\infty, \alpha \neq 1, \alpha>0
$$


and $h(x)$ is bounded in $\mathbb{R}$.

Definition 1.1 We will say that the Equation (1) has the Hyers-Ulam stability if there exists a positive constant $K>0$ with the following property:

For every $\varepsilon>0, z \in C^{2}[a, b]$, if

$$
\left|z^{\prime \prime}-F(x, z(x))\right| \leq \varepsilon
$$

with the initial condition (2), then there exists a solution $w(x) \in C^{2}[a, b]$ of the Equation (1), such that

$$
|z(x)-w(x)| \leq K \varepsilon .
$$

Definition 1.2 We say that Equation (3) has the HyersUlam stability with initial conditions (4) if there exists a positive constant $K>0$ with the following property:

For every $\varepsilon>0, z \in C^{2}[a, b]$, if

$$
\left.\left|z^{\prime \prime}-\right| z\right|^{\alpha} \operatorname{sgn} z \mid \leq \varepsilon
$$

and $z(a)=z^{\prime}(a)=0$, then there exists some $w \in C^{2}[a, b]$ satisfying $w^{\prime \prime}-|w|^{\alpha} \operatorname{sgn} w=0$ and $w(a)=w^{\prime}(a)=0$, such that $|z(x)-w(x)| \leq K \varepsilon$.

\section{Main Results on Hyers-Ulam Stability}

Theorem 2.1 If $z \in C^{2}[a, b]$ is such that

$$
|z(x)| \leq\left|z^{\prime}(x)\right| \text { and } A<\left(\max _{a \leq x \leq b}|z(x)|\right)^{1-\alpha},
$$

then the Equation (1) is stable in the sense of Hyers and Ulam.

Proof. Let $\varepsilon>0$ and $z$ be a twice continuously differentiable real-valued function on $I=[a, b]$. We will show that there exists a function $w(x) \in C^{2}(I)$ satisfying Equation (1) such that

$$
|z(x)-w(x)| \leq k \varepsilon
$$

where $k$ is a constant that never depends on $\varepsilon$ nor on $w(x)$. Since $z(x)$ is a continuous function on $I$, then it keep its sign on some interval $[a, x] \subseteq I$.

Without loss of generality assume that $z(x)>0$ on $[a, x]$. Assume that $\varepsilon>0, z \in C^{2}(I)$ satisfies the inequation (5) with the initial conditions (2) and that

$$
M=\max _{a \leq x \leq b}|z(x)| .
$$

From the inequality (5) we have

$$
-\varepsilon \leq z^{\prime \prime}-F(x, z(x)) \leq \varepsilon
$$

Since $z(x)>0$ on $[a, x] \subseteq I$ and $z(a)=0$, then by Mean Value Theorem $z^{\prime}>0$ in $(a, x)$. Multiplying the inequality (7) by $z^{\prime}>0$ and then integrating from $a$ to $x$, we obtain

$$
-2 \varepsilon z \leq z^{\prime 2}(x)-2 \int_{a}^{x} F(x, z(x)) z^{\prime} \mathrm{d} x \leq 2 \varepsilon z
$$

Since $|z(x)| \leq\left|z^{\prime}(x)\right|$ we get that

$$
z^{2}(x) \leq 2 \int_{a}^{x} F(x, z(x)) z^{\prime} \mathrm{d} x+2 \varepsilon z \leq A M^{\alpha-1} z^{2}+2 \varepsilon z
$$

Therefore

$$
\max _{a \leq x \leq b}|z(x)| \leq \frac{2 \varepsilon}{1-A M^{\alpha-1}}
$$

Hence $|z(x)| \leq k \varepsilon$, for all $x \geq a$. Obviously, $w_{0}(x)=0 \quad$ satisfies the Equation (1) and the zero initial condition (2) such that

$$
\left|z(x)-w_{0}(x)\right| \leq k \varepsilon
$$

Hence the Equation (1) has the Hyers-Ulam stability with initial condition (2).

Remark 2.1 Suppose that $z \in C^{2}(I)$ satisfies the inequality (5) with the initial condition (2). If

$$
\left|z^{\prime}(x)\right| \leq|z(x)|
$$

then, if

$$
A>\left(\max _{a \leq x \leq b}|z(x)|\right)^{1-\alpha}
$$

we can similarly show that the Equation (1) has the Hyers-Ulam stability with initial condition (2).

Theorem 2.2 Suppose that $z:[a, b] \rightarrow \mathbb{R}$ is a twice continuously differentiable function and $|z(x)| \leq\left|z^{\prime}(x)\right|$. If $B<\left(\max _{a \leq x \leq b}|z(x)|\right)^{1-\alpha}$ then the Equation (3) is stable in the sense of Hyers and Ulam.

Proof. Let $\varepsilon>0$ and $z$ be a twice continuously differentiable real-valued function on $I=[a, b]$. We will show that there exists a function $w(x) \in C^{2}(I)$ satisfying Equation (3) such that

$$
|z(x)-w(x)| \leq k \varepsilon
$$

where $k$ is a constant that never depends on $\varepsilon$ nor on $w(x)$. Since $z(x)$ is a continuous function on $I$ then it keeps its sign on some interval $[a, x] \subseteq I$. Without loss of generality assume that $z(x)>0$ on $[a, x]$.

Suppose that $\varepsilon>0, z \in C^{2}(I)$ satisfies the inequation (6) with the initial conditions (4) and that

$$
\begin{aligned}
& M=\max _{a \leq x \leq b}|z(x)| \text {. We have } \\
& \qquad-\varepsilon \leq z^{\prime \prime}-h(x) z^{\alpha} \leq \varepsilon
\end{aligned}
$$

Since $z^{\prime}>0$ in $(a, x)$ then, Multiplying the inequality (8) by $z^{\prime}$ and integrating, we obtain

$$
-2 \varepsilon z \leq z^{\prime 2}(x)-2 \int_{a}^{x} h(x) z^{\alpha} z^{\prime} \mathrm{d} x \leq 2 \varepsilon z
$$

By hypothesis $|z(x)| \leq\left|z^{\prime}(x)\right|$, so we get that 


$$
z^{2}(x) \leq 2 h\left(x^{*}\right) M^{\alpha-1} \int_{a}^{x} z \cdot z^{\prime} \mathrm{d} x+2 \varepsilon z \leq B M^{\alpha-1} z^{2}+2 \varepsilon z
$$

Therefore

$$
\max _{a \leq x \leq b}|z(x)| \leq \frac{2 \varepsilon}{1-B M^{\alpha-1}}
$$

Hence $|z(x)| \leq k \varepsilon$, for all $x \geq x_{0}$. Clearly, the zero function $w_{0}(x)=0$ satisfies the Equation (1) and the zero initial condition (2) such that

$$
\left|z(x)-w_{0}(x)\right| \leq k \varepsilon
$$

Hence the Equation (3) has the Hyers-Ulam stability with initial condition (4).

Remark 2.2 Suppose that $z \in C^{2}(I)$ satisfies the inequality (6) with the initial condition (4). If

$$
\left|z^{\prime}(x)\right| \leq|z(x)| \text { then, if } B>\left(\max _{a \leq x \leq b}|z(x)|\right)^{1-\alpha}
$$

we can similarly show that the Equation (3) has the Hyers-Ulam stability with initial condition (4).

Example 2.2 Consider the equation

$$
z^{\prime \prime}-\varepsilon^{2} \sin \left(\frac{x}{\varepsilon^{2}+1}\right) z^{\frac{1}{2}}=0
$$

and the inequality

$$
\left|z^{\prime \prime}-\varepsilon^{2} \sin \left(\frac{x}{\varepsilon^{2}+1}\right) z^{\frac{1}{2}}\right| \leq \varepsilon
$$

where $0 \leq x \leq 1$.

It should be noted that for a given $\varepsilon>0, z(x)=\varepsilon^{2} x$, satisfies the inequation (10) and the conditions of the Theorem 2.2. Therefore the Equation (9) has the HyersUlam stability.

\section{A Special Case of Equation (3)}

Consider the special case (when $\alpha=1$ ) of the Equation (3)

$$
z^{\prime \prime}-h(x) z=0
$$

with the initial conditions

$$
z(a)=z^{\prime}(a)=0
$$

and the inequation

$$
\left|z^{\prime \prime}-h(x) z\right| \leq \varepsilon
$$

where $z \in C^{2}(I), I=[a, b],-\infty<a<b<\infty$.

Theorem 3.1 Assume that $z:[a, b] \rightarrow \mathbb{R}$ is a twice continuously differentiable function and $|z(x)| \leq\left|z^{\prime}(x)\right|$ Then, If $B<1$ the Equation (11) is stable in the sense of Hyers and Ulam.

Proof. Assume that $\varepsilon>0$ and that $z$ is a twice continuously differentiable real-valued function on
$I=[a, b]$. We will show that there exists a function $w(x) \in C^{2}(I)$ satisfying Equation (11) such that

$$
|z(x)-w(x)| \leq k \varepsilon
$$

where $k$ is a constant that never depends on $\varepsilon$ nor on $w(x)$. Since $z(x)$ is a continuous function on $I$ then it keeps its sign on some interval $[a, x] \subseteq I$. Without loss of generality assume that $z(x)>0$ on $[a, x] \subseteq I$. Suppose that $\varepsilon>0, z \in C^{2}(I)$ satisfies the inequation (13) with the initial conditions (12).

We have

$$
-\varepsilon \leq z^{\prime \prime}-h(x) z \leq \varepsilon
$$

Applying the Mean Value Theorem to the function $z(x)$ on the interval $[a, x]$, we find that $z^{\prime}>0$ in $(a, x)$. Multiplying the inequality (14) by $z^{\prime}>0$ and then integrating we obtain

$$
-2 \varepsilon z \leq z^{\prime 2}(x)-2 \int_{a}^{x} h(x) z \cdot z^{\prime} \mathrm{d} x \leq 2 \varepsilon z
$$

If $|z(x)| \leq\left|z^{\prime}(x)\right|$, we obtain the inequality

$$
z^{2}(x) \leq 2 h\left(x^{*}\right) \int_{a}^{x} z \cdot z^{\prime} \mathrm{d} x+2 \varepsilon z \leq B z^{2}+2 \varepsilon z
$$

Therefore

$$
\max _{a \leq x \leq b}|z(x)| \leq \frac{2 \varepsilon}{1-B}
$$

Thus $|z(x)| \leq k \varepsilon$, for all $x \geq x_{0}$. The zero solution $w_{0}(x)=0$ of the Equation (11) with the zero initial condition (12) such that

$$
\left|z(x)-w_{0}(x)\right| \leq k \varepsilon
$$

Hence the Equation (11) has the Hyers-Ulam stability with initial condition (12).

Remark 3.1 Assume that $z \in C^{2}(I)$ satisfies the inequality (13) with the initial condition (12). If $\left|z^{\prime}(x)\right| \leq|z(x)|$ then, if $B>1$ we can similarly obtain the Hyers-Ulam stability criterion for the Equation (11) has with initial condition (12).

Remark 3.2 It should be noted that if $z<0$ on $(a, x]$ and $z(a)=0$, hence $z^{\prime}<0$ on $(a, x)$, then in the proofs of Theorem 2.1, 2.2 and 3.1, we can multiply by $z^{\prime}<0$ the inequation (7) (and (8), (14)) to obtain the inequality

$$
\varepsilon z^{\prime} \leq z^{\prime} \cdot z^{\prime \prime}-z^{\prime} F(x, z(x)) \leq-\varepsilon z^{\prime}
$$

Then we can apply the same argument used above to get sufficient criteria for the Hyers-Ulam stability for the Equations (1), (3) and (11).

Example 3.1 Consider the equation

$$
z^{\prime \prime}-\frac{1}{2} \cos \left(\frac{1}{x+1}\right) z=0
$$


and the inequality

$$
\left|z^{\prime \prime}-\frac{1}{2} \cos \left(\frac{1}{x+1}\right) z\right| \leq \varepsilon
$$

where $0 \leq x \leq 1$.

First it should be noted that for a given $\varepsilon>0$, $z(x)=\varepsilon x$, satisfies the inequation (16) and the conditions of the Theorem 3.1. Therefore the Equation (15) has the Hyers-Ulam stability.

\section{An Additional Case On Hyers-Ulam Stability}

In this section we consider the Hyers-Ulam stability of the following equation

$$
z^{\prime \prime}=\phi(x, z(x))
$$

with the initial condition

$$
z(a)=z^{\prime}(a)=0
$$

where

$$
z \in C^{2}(I), I=[a, b],-\infty<a<b<\infty
$$

and $\phi(x, z(x))$ is continuous for $x \in I, z \in \mathbb{R}$ such that

$$
|\phi(x, z(x))-\phi(x, w(x))| \leq L|z-w| .
$$

Using an argument similar to that used in [16], we can prove the following Theorem:

Theorem 4.1 Suppose that $z:[a, b] \rightarrow \mathbb{R}$ is a twice continuously differentiable function.

If $\frac{L(b-a)^{2}}{2}<1$, then the problem (17), (18) is stable in the Hyers-Ulam sense.

Proof. Let $\varepsilon>0$ and $z$ be a twice continuously differentiable real-valued function on $I=[a, b]$ satisfying the inequality

$$
-\varepsilon \leq z^{\prime \prime}-\phi(x, z(x)) \leq \varepsilon
$$

We will show that there exists a function

$$
w(x) \in C^{2}(I)
$$

Satisfying Equation (18) such that

$$
|z(x)-w(x)| \leq k \varepsilon
$$

where $k$ is a constant that doesn't depend on $\varepsilon$ nor on $w(x)$. If we integrate the inequality (19) with respect to $x$, we should obtain

$$
\begin{aligned}
& -\frac{\varepsilon(b-a)^{2}}{2} \leq-\frac{\varepsilon(x-a)^{2}}{2} \leq z-\int_{a}^{x} \int_{a}^{t} \phi(s, w(s)) \mathrm{d} s \\
& \leq \frac{\varepsilon(x-a)^{2}}{2} \leq \frac{\varepsilon(b-a)^{2}}{2}
\end{aligned}
$$

It is clear that $w(x)=\int_{a}^{x} \int_{a}^{t} \phi(s, w(s)) \mathrm{d} s$ is a solution of the Equation (21)

$$
w^{\prime \prime}=\phi(x, w(x))
$$

satisfying the zero initial condition

$$
w(a)=w^{\prime}(a)=0
$$

Now, let's estimate the difference

$$
\begin{aligned}
& |z(x)-w(x)| \leq\left|z-\int_{a}^{x} \int_{a}^{t} \phi(s, z(s)) \mathrm{d} s\right| \\
& +\left|\int_{a}^{x} \int_{a}^{t} \phi(s, w(s)) \mathrm{d} s-\int_{a}^{x} \int_{a}^{t} \phi(s, z(s)) \mathrm{d} s\right|
\end{aligned}
$$

Since the function $\phi(x, z(x))$ satisfies the Lipschitz condition, and from the inequality (20) we have

$$
|z(x)-w(x)| \leq \frac{\varepsilon(b-a)^{2}}{2}+L \frac{(b-a)^{2}}{2}|w(s)-z(s)|
$$

From which it follows that

$$
|z(x)-w(x)| \leq \frac{\varepsilon(b-a)^{2}}{2-L(b-a)^{2}}
$$

where $2>L(b-a)^{2}$. Hence the problem (17), (18) has the Hyers-Ulam stability.

Remark 4.1 Notice that if $\phi(x, z(x))=h(x)|z|^{\alpha}$, satisfies Lipschitz condition in the region $x \in I, z \in \mathbb{R}$, then the Emden-Fowler nonlinear differential equation $z^{\prime \prime}-h(x)|z|^{\alpha} \operatorname{sgn} z=0$ is Hyers-Ulam stable with zero initial condition.

\section{Acknowledgements}

The author thanks the anonymous referees for helpful comments and suggestions.

\section{REFERENCES}

[1] S. M. Ulam, "Problems in Modern Mathematics,” John Wiley \& Sons, New York, 1964.

[2] D. H. Hyers, "On the Stability of the Linear Functional Equation,” Proceedings of the National Academy of Sciences of the United States of America, Vol. 27, No. 4, 1941, pp. 222-224. doi:10.1073/pnas.27.4.222

[3] T. M. R assias, "On the Stability of the Linear Mapping in Banach Spaces," Proceedings of the American Mathemaical Society, Vol. 72, No. 2, 1978, pp. 297-300. doi:10.1090/S0002-9939-1978-0507327-1

[4] T. Miura, S.-E. Takahasi and H. Choda, "On the HyersUlam Stability of Real Continuous Function Valued Differentiable Map," Tokyo Journal of Mathematics, Vol. 24, No. 2, 2001, pp. 467-476. doi:10.3836/tjm/1255958187

[5] S. M. Jung, "On the Hyers-Ulam-Rassias Stability of Ap- 
proximately Additive Mappings,” Journal of Mathematics Analysis and Application, Vol. 204, No. 1, 1996, pp. 221-226. doi:10.1006/jmaa.1996.0433

[6] C. G. Park, "On the Stability of the Linear Mapping in Banach Modules," Journal of Mathematics Analysis and Application, Vol. 275, No. 2, 2002, pp. 711-720. doi:10.1016/S0022-247X(02)00386-4

[7] C. Alsina and R. Ger, "On Some Inequalities and Stability Results Related to the Exponential Function,” Journal of Inequalities and Application, Vol. 2, No. 4, 1998, pp. 373-380.

[8] E. Takahasi, T. Miura and S. Miyajima, "On the HyersUlam Stability of the Banach Space-Valued Differential Equation $y^{\prime}=\lambda y$," Bulletin of the Korean Mathematical Society, Vol. 39, No. 2, 2002, pp 309-315. doi:10.4134/BKMS.2002.39.2.309

[9] T. Miura, S. Miyajima and S.-E. Takahasi, "A Characterization of Hyers-Ulam Stability of First Order Linear Differential Operators," Journal of Mathematics Analysis and Application, Vol. 286, No. 1, 2003, pp. 136-146.

[10] S. M. Jung, "Hyers-Ulam Stability of Linear Differential Equations of First Order," Journal of Mathematics Analysis and Application, Vol. 311, No. 1, 2005, pp. 139-146. doi:10.1016/j.jmaa.2005.02.025

[11] G. Wang, M. Zhou and L. Sun, "Hyers-Ulam Stability of Linear Differential Equations of First Order," Applied Mathematics Letters, Vol. 21, No. 10, 2008, pp 10241028. doi:10.1016/j.aml.2007.10.020

[12] Y. Li, "Hyers-Ulam Stability of Linear Differential Equations," Thai Journal of Mathematics, Vol. 8, No 2, 2010 , pp. 215-219.

[13] Y. Li and Y. Shen, "Hyers-Ulam Stability of Nonhomogeneous Linear Differential Equations of Second Order," International Journal of Mathematics and Mathematical Sciences, Vol. 2009, 2009, Article ID: 576852, p 7.

[14] P. Gavruta, S. Jung and Y. Li, "Hyers-Ulam Stability for Second-Order Linear Differential Equations with Boundary Conditions," Electronic Journal of Differential Equations, Vol. 2011, No. 80, 2011, pp. 1-7.

[15] M. N. Qarawani, "Hyers-Ulam Stability of Linear and Nonlinear Differential Equations of Second Order," International Journal of Applied Mathematics, Vol. 1, No. 4, 2012, pp. 422-432.

[16] I. A. Rus, "Ulam Stability of Ordinary Differential Equations," Studia Universitatis Babeş-Bolyai-Series Mathematica, Vol. LIV, No. 4, 2009, pp. 125-133. 